\title{
Non-Hermitian adiabatic transport in spaces of exceptional points
}

\author{
J. Höller ${ }^{1}$, N. Read ${ }^{1,2}$, and J.G.E. Harris ${ }^{1,2}$ \\ ${ }^{1}$ Department of Physics, Yale University, P.O. Box 208120, New Haven, CT 06520-8120 \\ ${ }^{2}$ Department of Applied Physics, Yale University, P.O. Box 208284, New Haven, CT 06520-8284
}

(Dated: August 10, 2020)

\begin{abstract}
We consider the space of $n \times n$ non-Hermitian Hamiltonians $(n=2,3, \ldots)$ that are equivalent to a single $n \times n$ Jordan block. We focus on adiabatic transport around a closed path (i.e. a loop) within this space, in the limit as the time-scale $T=1 / \varepsilon$ taken to traverse the loop tends to infinity. We show that, for a certain class of loops and a choice of initial state, the state returns to itself and acquires a complex phase that is $\varepsilon^{-1}$ times an expansion in powers of $\varepsilon^{1 / n}$. The exponential of the term of $n$th order (which is equivalent to the "geometric" or Berry phase modulo $2 \pi$ ), is thus independent of $\varepsilon$ as $\varepsilon \rightarrow 0$; it depends only on the homotopy class of the loop and is an integer power of $e^{2 \pi i / n}$. One of the conditions under which these results hold is that the state being transported is, for all points on the loop, that of slowest decay.
\end{abstract}

\section{INTRODUCTION}

\section{A. Background}

The introduction of weak linear dissipation or amplification into a system of $n$ classical harmonic oscillators results in time evolution that can be described using an $n \times n$ Hamiltonian matrix $H$ that is non-Hermitian. The non-Hermiticity of $H$ gives rise to the familiar decay (or growth) of such a system's eigenstates (normal modes). It also opens the possibility of "exceptional points" (EPs) in parameter space, at which $H$ is not fully diagonalizable. In the neighborhood of an EP, the eigenvalues exhibit branch-point behavior as functions of the parameters, and so encircling it permutes the eigenvalues and eigenspaces [1 3], an effect referred to as flipping, monodromy, or spectral flow.

In recent years, EPs have been studied experimentally in a wide range of settings, including microwave [4, 5], electrical 6], optical [7], cavity QED [8], exciton [9], acoustic [10], and mechanical [11] systems. While each of these realizations has offered some degree of control over $H$, in most experiments the number $m$ of independent control parameters is insufficient to specify an arbitrary $H$. As a result, EPs are typically observed to occur at isolated points within the $m$-dimensional space of control parameters. In contrast, if we consider the space $M_{n}(\mathbf{C}) \cong \mathbf{C}^{n^{2}}$ of all $n \times n$ complex matrices $H$, then EPs are not isolated, but in fact form subspaces of $M_{n}(\mathbf{C})$ of dimension larger than zero [2, 12]. These subspaces are topological spaces (not vector spaces), and in the neighborhood of a generic point in such a subspace it is a smooth (indeed, complex analytic) manifold; we usually refer to these subspaces simply as spaces of EPs. One part of the following work is the description of the geometry and topology of these spaces in the simplest cases; we also explain how this is relevant to the topic of adiabatic evolution that we wish to study, and to which we now turn.

Leaving aside EPs for a moment, the evolution of a system under an asymptotically slow ("adiabatic") smooth variation of some parameters in time has been the subject of much study in both Hermitian and non-Hermitian cases. In what follows, we concentrate on evolution along a closed path (a loop) in parameter space [for example, $\left.M_{n}(\mathbf{C})\right]$. In Hermitian systems, the adiabatic theorem [13, 14] guarantees, in terms of the eigenstates of the "instantaneous" Hamiltonian at any point of the loop, that if the system is initially in an eigenstate (or in a subspace in Hilbert space of degenerate eigenvalues) and if the degeneracy of that eigenvalue does not change at any point during the evolution, then at the end of the adiabatic evolution the system will be found in the same eigenspace in which it started. Moreover, the phase of the state vector changes by an amount whose asymptotic form, as the time $T$ taken for the loop tends to infinity, has two leading contributions: the integral of the eigenvalue along the loop (the dynamical phase), which typically is linear in $T$; and the geometric or Berry phase, which is independent of $T$ [15]. The Berry phase modulo $2 \pi$, or phase factor, is the holonomy of a natural connection (i.e. "vector potential"), and may have further topological significance [15, 16]. (For evolution of a degenerate eigenspace, the phase factor becomes a unitary map [17].)

In contrast, in non-Hermitian systems there exists a mode or modes that are "dominant", meaning they have the largest rate of exponential growth (or the slowest decay). During adiabatic evolution along a generic loop the system tends to transition into one of these modes, which then dominates at long times, even when the eigenvalue (or its real or imaginary parts) of the chosen mode does not coincide with that of another mode at any point on the loop. In this situation the adiabatic theorem does not hold for all the modes, but only for the dominant one [18]. If a different mode becomes dominant somewhere along the loop, then even this statement breaks down. This occurs generically (because of monodromy, which was mentioned already) when the loop encircles an EP without passing through one; as a result, no adiabatic theorem applies for strict adiabatic transport around such a loop [19, 20]. We note that some of the experimental literature (e.g. Refs. [4, 5, 11]) is concerned instead with a "quasiadiabatic" limit of evolution that is slow, but not asymp- 
totically slow. In that regime, after evolution along a loop that encircles an EP, the system may end in a mode different from the one in which it began [19, 21], demonstrating the monodromy around the EP. We emphasize that this is not the same as the strict (i.e. asymptotically slow) adiabatic evolution that we consider in this paper.

\section{B. Overview of results}

In this paper, instead of adiabatic transport along a loop that never passes through any EP, we consider loops that lie entirely within one of the spaces of EPs that were mentioned already. The simplest example concerns a $2 \times 2$ non-Hermitian Hamiltonian matrix $H$; its EPs occur when $H$ is similar to a single $2 \times 2$ Jordan block. [We recall the Jordan canonical form: an arbitrary matrix can be transformed by change of basis (i.e. similarity transformation) into block diagonal form, where the diagonal blocks are Jordan blocks, and the other blocks are zero; in each Jordan block, the diagonal elements are all the same eigenvalue. The vectors in such a basis are termed generalized eigenvectors. The Jordan canonical form of the matrix is unique up to permutations of the blocks.] The eigenvalue on the diagonal in the Jordan block can be set to zero by adding a multiple of the identity; throughout our discussion, we will assume this is already done, as allowing it to be nonzero produces only very simple changes.

These EPs form a single space that we call $\mathcal{E} \mathcal{P}_{2}$, which is a subspace of $S M_{n}(\mathbf{C}) \subset M_{n}(\mathbf{C})$, the space of traceless complex $n \times n$ matrices, here with $n=2$ (we give details below); traceless $2 \times 2$ Hamiltonians not in $\mathcal{E P}_{2}$ are fully diagonalizable. This example has simple geometry and is quite tractable. As it can be readily generalized to the case of traceless $n \times n$ Hamiltonians similar to a single $n \times n$ Jordan block $J_{n}$,

$$
J_{n}=\left(\begin{array}{lllll}
0 & 1 & & & \\
& 0 & 1 & & \\
& & & \ddots & \\
& & & & 1 \\
& & & & 0
\end{array}\right),
$$

and with similar results for all $n>1$, we carry out the analysis in this more general case. For each $n$, we denote the space of these EPs by $\mathcal{E} \mathcal{P}_{n} \subset S M_{n}(\mathbf{C})$. For $n>2$, there are also EPs in $S M_{n}(\mathbf{C})$, but not in $\mathcal{E P}_{n}$, at which other Jordan block structures arise, and the situation becomes much more complex. We do not consider those cases in the present paper.

We first describe the geometry of the space $\mathcal{E P}_{n}$, with particular emphasis on the simplest case, $n=2$. We find in particular that these spaces are $n$-fold connected (doubly connected for $n=2$ ), in the sense that there are closed paths (loops) lying in $\mathcal{E P}_{n}$ that cannot be contracted (within $\mathcal{E P}_{n}$ ) to a point, but traversing such a loop $n$ times produces a loop that can be so contracted.
In other words, one may associate a winding number (defined modulo $n$ ) to any loop, and concatenation of two loops that both begin and end at the same point gives a loop whose winding number is the sum of those of the two given loops, modulo $n$.

Then, as for the usual adiabatic theorems, we consider a loop in $\mathcal{E P}_{n}$, parametrized by Hamiltonians $H(s)$ for $0 \leq s \leq 1$, where $H(1)=H(0)$, and evolve the system in time $t$ with $s=t / T$; finally the asymptotics as $T \rightarrow \infty$ are studied, with the loop $H(s)$ fixed (independent of $T$ ). We find that the result of adiabatic transport in $\mathcal{E P}_{n}$ has features in common with the case of a non-degenerate eigenstate in a Hermitian system, but also substantial differences. For a class of loops and a choice of initial state vector (to be described in a moment), we find that as $T \rightarrow \infty$ the state vector returns to itself, multiplied by a complex number whose logarithm has the Puiseux series form

$$
\sum_{r=1}^{n} T^{1-r / n} \int_{0}^{1} d s a_{r}(s)
$$

(plus terms higher order in $1 / T^{1 / n}$ ), where $a_{r}(s)$ are complex functions that can be calculated from $H(s)$. Thus the (complex) dynamical phase (the terms with $r<n$ ) includes fractional powers of $T^{-1}$ (of which the $r=1$ term at least has been noticed previously [22]). The term of order $T^{0}$ (the $r=n$ term) is the geometric or Berry phase, and is only well-defined modulo $2 \pi i$. Remarkably, the exponential of this term is again the holonomy of a connection, and is precisely $e^{2 \pi i / n}$ raised to the power of the winding number of the loop in $\mathcal{E P}_{n}$; it is invariant under small deformations of the loop within that space.

To describe the conditions under which this result holds, it is useful to change basis in the evolution equation to the basis of instantaneous generalized eigenvectors of $H(s)$. In this basis, the effective Hamiltonian describing evolution is $H^{\prime}=J_{n}+T^{-1} A(s)$, where $A(s)$ is the adiabatic (Berry) connection matrix along the loop at $s$ (details appear below). The most generic case is that in which the lower-left element $A_{n 1}$ of $A$ is nonzero, and then $H^{\prime}$ is diagonalizable. For adiabatic transport in $\mathcal{E P}_{n}$, it is $H^{\prime}$ (rather than $H$ ) that determines the dominant mode. We assume that the same mode remains dominant everywhere on the loop, which is ensured if $A_{n 1}$ does not touch the negative real axis or zero. Our result holds for such loops and when the initial state vector is this dominant mode.

In addition, we identify an important subclass of such loops for which adiabatic transport of any eigenstate of $J_{n}+T^{-1} A$ stays in that state for all time. This consists of loops for which $A(s)=A$ is independent of $s$. As long as $A_{n 1}$ is not zero, such a "straight" loop in $\mathcal{E P}_{n}$ produces the form (2) for each eigenstate, not only for the dominant one (the coefficients $a_{r}$ for $r \neq n$ differ for each eigenstate, however). Loops of this form that possess non-zero winding number exist in $\mathcal{E P}_{n}$, and the holonomy (Berry phase factor) of such a loop is independent of which eigenstate of $J_{n}+T^{-1} A$ is transported. 
We also explain how the effects can be studied experimentally for $n=2$ in two implementations, including one described in Ref. 12.

In Sec. II , all the results just outlined are derived step by step, except for some parts that can be skipped without significant loss of understanding and which are relegated to the Appendices. Particular emphasis is placed on the simplest case, $n=2$, which we use to give examples. In Sec.III, we describe the experimental implementations. Sec. IV is the Conclusion.

\section{DERIVATIONS OF RESULTS}

\section{A. Geometry of $\mathcal{E} \mathcal{P}_{n}$}

To obtain the results outlined in Sec. IB, it is convenient to use the evolution (Schrödinger) equation in the form $\partial_{t}|\psi\rangle=H|\psi\rangle$, so $i H$ would be the usual Hamiltonian of quantum mechanics. We treat $H$ as a matrix, and $|\psi\rangle$ stands for a column vector. Adding a multiple of the identity $I$ to a Hamiltonian merely shifts all the eigenvalues by the same amount, so we can assume that the trace of $H$ is zero. An $n \times n$ complex Hamiltonian that is similar to a single Jordan block with eigenvalue zero, meaning that $H=\Lambda J_{n} \Lambda^{-1}$ where $\Lambda$ is an invertible complex invertible matrix, is said to lie at an EP of type $E P_{n}$. (Note that the columns of $\Lambda$ are generalized eigenvectors of $H$; we denote them by $\left|u_{i}\right\rangle$ for $i=1, \ldots$, n.)

To describe the geometry of the space $\mathcal{E} \mathcal{P}_{n}$ of all such $H$, first notice that the matrices that commute with $J_{n}$ have the form $a I+b_{1} J_{n}+\cdots+b_{n-1} J_{n}^{n-1}$, where $I$ is the identity, and $a, b_{i}$ are complex numbers. Such matrices with $a \neq 0$ form a Lie group $\mathbf{C}^{\times} \times \mathcal{J}_{n}$, which is a subgroup of $G L_{n}(\mathbf{C})$, the group of invertible $n \times n$ complex matrices. Here $\mathbf{C}^{\times}$is the group of nonzero complex numbers (under multiplication), and $\mathcal{J}_{n}$ is the group of $n \times n$ matrices of the form $I+b_{1}^{\prime} J_{n}+\cdots+b_{n-1}^{\prime} J_{n}^{n-1}$, where $b_{i}^{\prime}$ are complex numbers. Then $\mathcal{E P}_{n}$ can be identified as the quotient space $\mathcal{E P}_{n} \cong G L_{n}(\mathbf{C}) /\left[\mathbf{C}^{\times} \times \mathcal{J}_{n}\right]$; see Appendix $\mathrm{A}$ for further details. It is a non-compact space of complex dimension $n(n-1)$, but has a "deformation retract" 23] onto $S U(n) / \mathbf{Z}_{n}$, that is the group $S U(n)$ of unitary matrices of determinant 1 , modulo its center $\mathbf{Z}_{n}$, the cyclic group of order $n$. The fundamental group of this space is $\pi_{1}\left(\mathcal{E} \mathcal{P}_{n}\right) \cong \mathbf{Z}_{n}$ (see Appendix A). That means that loops in the space can be characterized (modulo small deformations) by a single winding number defined modulo $n$, as described in Sec. IB. We note that if we allowed the single eigenvalue of $H$ to be nonzero instead of requiring it to be zero, then the space of such Hamiltonians would be $\cong \mathcal{E} \mathcal{P}_{n} \times \mathbf{C}$ and have complex dimension higher by 1 , but the fundamental group would be unchanged.

\section{Example: $n=2$}

As illustration, for $n=2$, we can more explicitly describe traceless Hamiltonians as

$$
H=\left(\begin{array}{cc}
Z & X-i Y \\
X+i Y & -Z
\end{array}\right)
$$

where $X, Y, Z$ are complex numbers. With our conventions, $i H$ would be Hermitian (with respect to the standard inner product) if $X, Y, Z$ were all imaginary. However, for general non-Hermitian $H$, an inner product plays no essential role, and we avoid using one on $\mathbf{C}^{n}$ at any stage. For $n=2$, clearly there are no exceptional points other than those in $\mathcal{E} \mathcal{P}_{2}$. If $\mathbf{X}=(X, Y, Z)^{T}$ (the superscript $T$ denotes transpose), then $H$ is in $\mathcal{E} \mathcal{P}_{2}$ if and only if $|\operatorname{Re} \mathbf{X}|=|\operatorname{Im} \mathbf{X}|>0$ and $\operatorname{Re} \mathbf{X} \cdot \operatorname{Im} \mathbf{X}=0$ [2] (here the standard inner product and norm on $\mathbf{R}^{3}$ were used). If we fix $|\operatorname{Re} \mathbf{X}|$ to 1 , then because an ordered pair of orthogonal unit vectors in $\mathbf{R}^{3}$ (such as $\operatorname{Re} \mathbf{X}, \operatorname{Im} \mathbf{X}$ ) determines an orthonormal basis with positive orientation in $\mathbf{R}^{3}$, the space of such pairs forms the special orthogonal group in three dimensions, or real projective 3 -space, $S O(3) \cong \mathbf{R P}^{3} \cong S U(2) / \mathbf{Z}_{2}$. It is well-known that the fundamental group of this space is $\pi_{1} \cong \mathbf{Z}_{2}$ (i.e. it is doubly connected; see Sec. [B] [23]. Then $\mathcal{E P}_{2} \cong S O(3) \times \mathbf{R}$, where the second factor represents $\ln |\operatorname{Re} \mathbf{X}|$ and is contractible. Hence $\mathcal{E} \mathcal{P}_{2}$ is doubly connected also, that is $\pi_{1}\left(\mathcal{E} \mathcal{P}_{2}\right)=\mathbf{Z}_{2}$.

\section{B. Adiabatic transport in $\mathcal{E P}_{n}$}

\section{General statements}

Now we consider adiabatic transport in $\mathcal{E P}_{n}$ for general $n$. We choose a smooth loop in $\mathcal{E P}_{n}$, so we have $H=H(s)$, a smooth function of $s \in[0,1]$ with $H(1)=$ $H(0)$ and $H \in \mathcal{E P}_{n}$ for all $s$. We evolve the system in time $t$ from 0 to $T>0$ with the time-dependent Hamiltonian $H=H(s=t / T)$ as in the usual adiabatic evolution. If we express the evolution equation $\partial_{t}|\psi\rangle=H|\psi\rangle$ in a basis of generalized eigenvectors $\left|u_{i}(s)\right\rangle$ of $H(s)=\Lambda(s) J_{n} \Lambda(s)^{-1}$ at each $s$ (that varies smoothly with $s$ ) then, for the column vector $|u\rangle=\Lambda^{-1}|\psi\rangle$ of components in this basis, it takes the form

$$
\varepsilon \partial_{s}|u\rangle=\left(J_{n}+\varepsilon A\right)|u\rangle,
$$

where $\varepsilon=1 / T$ and $A_{i j}=-\left\langle u_{i} \mid \partial_{s} u_{j}\right\rangle$ (i.e. $A=$ $\left.-\Lambda^{-1} \partial_{s} \Lambda\right)$ is the Berry connection evaluated on the tangent vector to the loop. Here the bras $\left\langle u_{i}(s)\right|$ are a smooth basis set of row vectors dual to the basis of kets $\left|u_{i}(s)\right\rangle$, so $\left\langle u_{i}(s) \mid u_{j}(s)\right\rangle=\delta_{i j}$ for each $s$ (this is not a use of an inner product); they are rows of $\Lambda^{-1}$. To keep later arguments simpler, we assume without loss of generality that $\Lambda(s)$ is periodic $[\Lambda(1)=\Lambda(0)]$, and so also $A(1)=A(0)$. There is a residual gauge freedom when 
we obtain eq. (4): the form is preserved under a further differentiable periodic $s$-dependent change of basis by $\widetilde{\Lambda}(s) \in \mathbf{C}^{\times} \times \mathcal{J}_{n}$ for all $s$ (see Appendix $\mathbf{B}$ ).

The key point now is that while $J_{n}$ is not diagonalizable, $J_{n}+\varepsilon A$ often is. As $\varepsilon \rightarrow 0$, $\operatorname{det}\left(J_{n}+\varepsilon A\right)=$ $(-1)^{n-1} \varepsilon A_{0}+\mathcal{O}\left(\varepsilon^{2}\right)$, where we write $A_{0}=A_{n 1}(s)$ and we assume henceforth that $A_{0}$ is nonzero for all $s$. From the characteristic equation, we find that the $s$-dependent eigenvalues of $J_{n}+\varepsilon A$ are $\lambda_{\mu}=\zeta^{\mu}\left(\varepsilon A_{0}\right)^{1 / n}$ $(\mu=0,1, \ldots, n-1)$ to leading order in $\varepsilon$, where $\zeta=e^{2 \pi i / n}$ (see Appendix C). Here we choose one $n$th root of $A_{0}$, which we take to be the principal branch, for which $\arg A_{0}^{1 / n} \in(-\pi / n, \pi / n]$, and denote it $A_{0}^{1 / n}$, and $\left(\varepsilon A_{0}\right)^{1 / n}=\varepsilon^{1 / n} A_{0}^{1 / n}\left(\varepsilon^{1 / n}>0\right)$. Solving iteratively for each eigenvalue, we can obtain series expansions

$$
\lambda_{\mu}=\sum_{r=1}^{\infty} a_{r} \varepsilon^{r / n} \zeta^{\mu r}
$$

with nonzero radius of convergence; such an expansion is called a Puiseux expansion. Note that here the coefficients $a_{r}=a_{r}(s)\left(a_{1}=A_{0}^{1 / n}\right)$ are independent of $\mu$ (because $\zeta^{\mu r}$ has been extracted), because if such an expansion satisfies the characteristic equation for one value $\mu$, then it does so for all $\mu$. Then as $\sum_{\mu} \lambda_{\mu}=\operatorname{tr}\left(J_{n}+\varepsilon A\right)=$ $\varepsilon \operatorname{tr} A$, we find that

$$
a_{n}=\frac{1}{n} \operatorname{tr} A
$$

and $a_{2 n}=a_{3 n}=\cdots=0$. If $A_{0}=0$ for some $s$ (contrary to our assumption), then the remaining elements of $A$ become important as $\varepsilon \rightarrow 0$, and there are different cases to study; we do not consider these in this paper.

If $A_{0} \neq 0$ makes a circuit $k$ times around the origin (say, as $s$ varies), then following the eigenvalues $\lambda_{\mu}$ continuously along the circuit produces a net cyclic permutation $\mu \rightarrow \mu+k(\bmod n)$. This monodromy of the eigenvalues has the same form as that we mentioned [1] in the first paragraph of Sec. [1 The situations are related because $\varepsilon A$ can be considered as a perturbation from the exceptional point $H=J_{n}$ to an "effective" Hamiltonian $H^{\prime}=J_{n}+\varepsilon A$, and we are considering a case in which the degeneracy of eigenvalues is fully lifted. Henceforth we assume that $A_{0}$ does not encircle the origin as $s$ varies from 0 to 1 .

If, in the adiabatic limit $\varepsilon \rightarrow 0$ and under our assumptions, a state prepared in the $\mu$ th instantaneous eigenvector of $J_{n}+\varepsilon A$ stayed in the corresponding eigenstate until $s=1$, then there would be a change in its "phase" (i.e. the log of the amplitude, which here is complex) of

$$
\sum_{r=1}^{n} \varepsilon^{r / n-1} \zeta^{\mu r} \int_{0}^{1} d s a_{r}(s)
$$

plus order $\varepsilon^{1 / n}$, plus possibly a further contribution to the geometric phase, which we discuss in Sec. II B3 below. Apart from the $r=n$ term, the terms displayed in expression (7) are dynamical phases, which depend on $T$. In addition to the usual one that is of order $T$ (absent here because we subtracted off the trace of $H$ ), there are also fractional powers of $T$ 22]. These may be considered "stretched exponential" dependence on the time scale $T$ of adiabatic evolution.

In view of our choice that $\Lambda(1)=\Lambda(0)$, the final $r=n$ term in (7) is a geometric phase, like the usual Berry phase in the case of non-degenerate eigenvalues, but given by the average $\int d s n^{-1} \operatorname{tr} A$ of the diagonal elements of $A$. It can change by a multiple of $2 \pi i$ under a "large" residual gauge transformation that winds in $\mathbf{C}^{\times}$as a function of $s$ (see Appendix $(\mathrm{B})$; thus it is well-defined only modulo $2 \pi i$. In other words, it is the Berry phase factor or holonomy

$$
e^{n^{-1} \int_{0}^{1} \operatorname{tr} A}
$$

that is well-defined if we do not keep track of the choice of basis along the path (i.e. is fully gauge invariant). The dynamical phase terms $r<n$ are gauge invariant (again, see Appendix [B]; these gauge-invariance properties are similar to the usual Hermitian case.

\section{A special class of loops}

An important special case of adiabatic transport in $\mathcal{E} \mathcal{P}_{n}$ is that in which $A$ (and hence $a_{r}$ ) is independent of $s$ (for all $r$ ). In that case, the coefficients in eq. (4) are constant, so the system does stay in an initial eigenstate of $J_{n}+\varepsilon A$ for all $s$ if it is in one initially, and the preceding remarks conclude the calculation. We note that the corresponding path is "straight" in $G L_{n}(\mathbf{C})$, with $\Lambda(s)=\Lambda(0) \exp (-s A)$, and that such paths can return to the starting point, so $\Lambda(1)=\Lambda(0)$. Moreover, these loops can be non-trivial in both $\pi_{1}\left(G L_{n}(\mathbf{C})\right)$ and $\pi_{1}\left(\mathcal{E} \mathcal{P}_{n}\right)$, that is, they can have non-zero winding number (modulo $n$ ) when projected to $\mathcal{E P}_{n}$. In the general case, in which $A$ is not constant, there could be further contributions to the geometric phase of the same order, which we discuss next.

\section{More general loops}

In order to examine the general scenario, we apply the adiabatic theorem to $J_{n}+\varepsilon A$. At leading order, the $\mu$ th eigenvector of $J_{n}+\varepsilon A$ can be chosen to be

$$
\left|v_{\mu}\right\rangle=\left(\begin{array}{c}
1 \\
\left(\varepsilon A_{0}\right)^{1 / n} \zeta^{\mu} \\
\cdot \\
\cdot \\
\left(\varepsilon A_{0}\right)^{(n-1) / n} \zeta^{\mu(n-1)}
\end{array}\right)\left[1+\mathcal{O}\left(\varepsilon^{1 / n}\right)\right]
$$

which is periodic in $s$ under our assumptions. If we use these instantaneous eigenvectors as a basis set (together 
with a dual basis as before), then in this basis the evolution equation becomes

$$
\varepsilon \partial_{s}|v\rangle=\left(D+\varepsilon A^{\prime}\right)|v\rangle
$$

where $D=\operatorname{diag}\left(\lambda_{0}, \ldots, \lambda_{n-1}\right)$, and $A_{\mu \nu}^{\prime}=-\left\langle v_{\mu} \mid \partial_{s} v_{\nu}\right\rangle$. Now we use the adiabatic theorem for this non-Hermitian non-degenerate situation [18]. As mentioned already, in this case, with eigenvalues $\lambda_{\mu}$ with differences much larger than $\varepsilon$ as $\varepsilon \rightarrow 0$, the adiabatic theorem in general does not hold for all the eigenspaces of $D$, but only for the dominant mode (the one with the largest real part of its eigenvalue). (In the less general case in which a permutation of the $\left|v_{\mu}\right\rangle \mathrm{s}$ makes $A^{\prime}$ block diagonal with the same block structure for all $s$, then the adiabatic theorem holds for the dominant mode in each block.) If we make the stronger assumption that $\left|\arg A_{0}(s)\right|<\pi$ for all $s$ (i.e. $A_{0}$ does not touch or cross the negative real axis) then, for all $s$ and as $\varepsilon \rightarrow 0, \operatorname{Re} \lambda_{\mu}$ is largest when $\mu=0$, and is non-degenerate. [Technically, we assume that $A_{0}(s)$ does not approach the negative real axis or zero closer than some small constant, say $\delta>0$.] With this assumption, it is not difficult to show that if the initial state is purely the dominant mode, then it remains in it for all $s$ with sufficient accuracy as $\varepsilon \rightarrow 0$ (see Ref. [18] and Appendix D). Moreover, the additional contribution to the geometric or Berry "phase" is found by integrating the diagonal element $A_{00}^{\prime}$ for the dominant mode.

In the present case, we find that

$$
A_{\mu \nu}^{\prime}=-\sum_{r=1}^{n-1} \frac{r}{n^{2}} \zeta^{(\nu-\mu) r} \frac{\partial_{s} A_{0}}{A_{0}}+\mathcal{O}\left(\varepsilon^{1 / n}\right),
$$

so the diagonal elements are given by $\frac{1-n}{2 n} \partial_{s} \ln A_{0}$ to leading order. Integrating from $s=0$ to 1 , the change in the complex amplitude is $\left[A_{0}(0) / A_{0}(1)\right]^{(n-1) /(2 n)}$. Because $A_{0}$ does not make a circuit around the origin, this factor is 1 . Then the net phase change through order $\varepsilon^{0}$ is given by the integrated Puiseux expansion (7). It is remarkable that, for adiabatic transport in $\mathcal{E} \mathcal{P}_{n}$, there is an order $\varepsilon^{0}$ part of $A^{\prime}$, yet this part still does not contribute to the geometric phase.

\section{Calculation of net holonomy}

Finally, we need to calculate the net geometric phase for a loop. First, because $\Lambda(1)=\Lambda(0)$, the nonAbelian holonomy [which is an element of $G L_{n}(\mathbf{C})$ ] is $\mathcal{P} \exp \int_{0}^{1} A d s(\mathcal{P}$ exp is path-ordering of the exponential). Hence the holonomy for any loop is $\Lambda(1)^{-1} \Lambda(0)=I$, and by considering a contractible loop and using Stokes's theorem, it follows that the non-Abelian Berry connection $A$ has zero (Yang-Mills) curvature.

The trace of $A$ is in the Lie subalgebra $g l_{1}(\mathbf{C}) \cong \mathbf{C}$ of $g l_{n}(\mathbf{C})$, so the corresponding Abelian holonomy is $\operatorname{det} \Lambda(0) / \operatorname{det} \Lambda(1)=1$ in $G L_{1}(\mathbf{C}) \cong \mathbf{C}^{\times}$. Then $n^{-1} \operatorname{tr} A$, which determines the geometric phase, is again a flat connection (i.e. its Berry curvature is zero). Its holonomy is

$$
e^{n^{-1} \int_{0}^{1} \operatorname{tr} A d s}=[\operatorname{det} \Lambda(0) / \operatorname{det} \Lambda(1)]^{1 / n}
$$

[with the $n$th root defined by imposing continuity on $\left.\{\operatorname{det} \Lambda(s)\}^{1 / n}\right]$; because of the vanishing curvature, it depends only on the homotopy class of the loop. For any contractible loop in $G L_{n}(\mathbf{C})$ this holonomy is 1 , but for a non-contractible loop it is an $n$th root of 1 , and so a power of $\zeta$. [Note that $\pi_{1}\left(G L_{n}(\mathbf{C})\right) \cong \mathbf{Z}$, so such loops exist; they are associated with the non-contractibility of $\mathbf{C}^{\times}$.] It is precisely $\zeta^{-1}$ to the power of the winding number of the path of $\operatorname{det} \Lambda(s)$ around the origin in $\mathbf{C}^{\times}$; note that this winding number changes by a multiple of $n$ under a residual gauge transformation. In $\mathcal{E} \mathcal{P}_{n}$, the fundamental group is $\mathbf{Z}_{n}$, so repeating a given loop $n$ times produces a loop that is contractible in $\mathcal{E} \mathcal{P}_{n}$, and hence the $n$th power of the holonomy for any loop must be 1 , consistent with our conclusion that the holonomy is a power of $\zeta$. This concludes the derivation of the general results. In Appendix A, we also explain that the mapping from a loop in $\mathcal{E} \mathcal{P}_{n}$ to a power of $\zeta$, given by the holonomy, Eq. (12), can be viewed as a torsion first Chern class $c_{1}$.

\section{Example: $n=2$}

As an illustration of the general results, we solve the $n=2$ model explicitly for $A$ constant and $A_{0} \neq 0$. An example of a non-contractible loop in $\mathcal{E} \mathcal{P}_{2}$ is parametrized by $\mathbf{X}(s)=(i \cos \phi(s), i \sin \phi(s), 1)^{T}$ with $\phi(s)=2 \pi s$. Then with $\left|u_{1}\right\rangle=\left(1, i e^{i \phi}\right)^{T}$ and $\left|u_{2}\right\rangle=(1,0)^{T}$, we find

$$
A=\left(\begin{array}{cc}
-2 \pi i & 0 \\
2 \pi i & 0
\end{array}\right)
$$

so $A_{0}=2 \pi i$, and the eigenvalues of $J_{2}+\varepsilon A$ are

$$
\begin{aligned}
\lambda_{0,1} & =-i \pi \varepsilon \pm \sqrt{2 \pi i \varepsilon-\pi^{2} \varepsilon^{2}} \\
& = \pm \pi^{1 / 2}(1+i) \varepsilon^{1 / 2}-i \pi \varepsilon+\mathcal{O}\left(\varepsilon^{3 / 2}\right) .
\end{aligned}
$$

The two leading terms agree with $a_{1}=A_{0}^{1 / 2}$ and $a_{2}=$ $\frac{1}{2} \operatorname{tr} A=-i \pi$, and further $a_{2 k}=0$ for $k>1$; note that the Berry phase is $\pi$ (modulo $2 \pi$ ). Our general theory tells us that these results are independent of the choice of gauge, and that the Berry phase is invariant under sufficiently slowly varying smooth changes in the loop. More generally, if we choose $\phi(s)=2 \pi m s$ for an integer $m$, giving a loop of winding number $m$, then the holonomy is $(-1)^{m}$.

\section{EXPERIMENTAL IMPLEMENTATIONS FOR $n=2$}

The simplest demonstration of our results is for nonHermitian $2 \times 2$ Hamiltonians, which are of the general 
form in Eq. (3). Here, we discuss two experimental setups which realize such a Hamiltonian, and we propose noncontractible loops along which adiabatic transport would produce the results in Eq. (15). In either setup, this means that if the system were initialized in the dominant mode, there would be a contribution to the (complex) dynamical phase of $(1+i) \sqrt{\pi T}$, and the Berry phase factor would be -1 .

The first setup consists of a qubit (two-level system) that is coupled to a waveguide, and which can decay to a third level. When the system is post-selected for evolution that remains within the qubit's Hilbert space, the resulting dynamics can be described via an effective Hamiltonian that is non-Hermitian, as demonstrated experimentally in Ref. [24]. In the rotating frame and rotating wave approximation, we identify the three complex numbers $X, Y$, and $Z$ in Eq. (3) with experimental parameters: $X=J \cos (\phi), Y=J \sin (\phi), Z=\Delta / 2-i \gamma / 4$, where $\Delta$ is the detuning of the drive applied to the qubit (via the waveguide), $\gamma$ the difference in decay rates of the two qubit states, $J$ the coupling strength (also known as Rabi frequency), and $\phi$ the (Rabi) phase. From the discussion below Eq. (3), we infer that exceptional points lie at $\Delta=0$ and $J=|\gamma| / 4$ for all $\alpha$. For fixed $\Delta=0$ and $J=|\gamma| / 4$, varying $\phi$ by $2 \pi$ describes a noncontractible loop. If $\gamma$ is also controlled [24], then the accessible subspace of $\mathcal{E} \mathcal{P}_{2}$ forms a frustum - a cone without its apex (because there, $H=0$ and so is diagonalizable).

Second, we argue that full control over Eq. (3) can be achieved with the optomechanical device used in Ref. [12]. The device consists of a dielectric membrane in the middle of an optical cavity. The membrane's vibrational modes can be coupled to each other by sending light at particular frequencies into the cavity; in addition to the complex-valued mutual coupling, light also introduces a complex-valued self-coupling term to each oscillating normal mode [25]. In Ref. [12], two pairs of light beams were used to couple two oscillating normal modes. It is straightforward to show that various combinations of laser tones (i.e. their power and detuning) can be chosen to give independent control over the complex parameters $X, Y$, and $Z$ in Eq. (3).

\section{CONCLUSION}

To conclude, we studied adiabatic transport around a loop in a space of exceptional points of type $E P_{n}$ for $n \times$ $n$ Hamiltonian matrices. We found that the dynamical phase is given by a Puiseux series of fractional powers of $T$, and that the Berry phase (modulo $2 \pi$ ) depends only on the homotopy class of the loop. The results hold for a choice of initial state that depends on both the loop (which must be in a certain class of loops), and on $T$.

Clearly, it would be of interest to carry out an analysis by similar methods in other cases, such as when $A_{0}=0$ all along the loop. Alternatively, for $n>2$ we can also consider, for example, spaces of EPs of types
$E P_{n^{\prime}}$ for $n^{\prime}<n$ within $M_{n}(\mathbf{C})$, or spaces of Hamiltonians for which the Jordan canonical form is a direct sum of several Jordan blocks with the same eigenvalue. We expect these cases to involve the non-Abelian connection for transporting a proper subspace [17], as well as effects similar to those we found for a Jordan block of size $n$. We leave these cases for later study.

\section{ACKNOWLEDGMENTS}

We are grateful for discussions with A. Alexandradinata and G. Moore. We acknowledge support from Yale University (JH), from AFOSR grant FA9550-15-1-0270 and ONR MURI N00014-15-1-2761 (JGEH), and (while the paper was under revision) from NSF grant no. DMR1724923 (JH and NR).

\section{Appendix A: Geometry and topology of $\mathcal{E P}_{n}$}

In this Appendix, we consider the geometry and topology of the space $\mathcal{E} \mathcal{P}_{n}$. We first show that it is a complex manifold of complex dimension $n(n-1)$, and has the same topology (i.e. what is called homotopy type [23]) as $S U(n) / \mathbf{Z}_{n}$ [for $n=2$, this becomes $S O(3)$, the space of real $3 \times 3$ orthogonal matrices of determinant 1]. This space is connected but not simply connected; its fundamental group is $\pi_{1}\left(\mathcal{E} \mathcal{P}_{n}\right) \cong \mathbf{Z}_{n}$, which is not the trivial group when $n \geq 2$, and corresponds to the $n$-fold connectedness described in Sec. IB. (The calculation in the main text is required in order to determine the Berry phase factor, relate it to a holonomy, show that there are loops for which it is not unity, and relate it to the winding number of the loop.) Finally, we also determine the low-dimensional homology and cohomology groups of $\mathcal{E} \mathcal{P}_{n}$ with integer coefficients, including the second cohomology group $H^{2}\left(\mathcal{E} \mathcal{P}_{n}\right)$, which is again $\cong \mathbf{Z}_{n}$. The latter group tells us about the possible holonomy for a loop in $\mathcal{E P}_{n}$, and we explain how the results in the text can be viewed as an example of a torsion first Chern class, which is an element of $\left.H^{2}(\mathcal{E P})_{n}\right)$.

To begin, for $H=\Lambda J_{n} \Lambda^{-1}$ and $\Lambda$ an invertible complex matrix [an element of the general linear group $G L_{n}(\mathbf{C})$ ], we see that multiplying $\Lambda$ on the right by any invertible matrix that commutes with $J_{n}$ produces the same $H$. The latter matrices form the subgroup $\mathbf{C}^{\times} \times \mathcal{J}_{n}$ defined in Sec. IIA It follows that the space of such $H$ is $\mathcal{E} \mathcal{P}_{n} \cong G L_{n}(\mathbf{C}) /\left[\mathbf{C}^{\times} \times \mathcal{J}_{n}\right]$, which is thus a homogeneous complex manifold. The complex dimension of $G L_{n}(\mathbf{C})$ is $n^{2}$, and that of $\mathbf{C}^{\times} \times \mathcal{J}_{n}$ is $n$, so the complex dimension of $\mathcal{E} \mathcal{P}_{n}$ is $n(n-1)$. This number can also be understood heuristically as follows: $M_{n}(\mathbf{C})$ has dimension $n^{2}$, and a generic matrix has $n$ distinct complex eigenvalues. The condition that all eigenvalues be zero imposes $n$ constraints, leaving an $n^{2}-n$ dimensional space. This space contains points not in $\mathcal{E} \mathcal{P}_{n}$, but it turns out that those points form subspaces of dimension $<n^{2}-n$; for exam- 
ple, for any $n \geq 2$ the space includes the zero matrix, which is not similar to any non-zero matrix.

To study further the geometry and topology of $\mathcal{E P}_{n}$, a useful first step is to analyze that of $G L_{n}(\mathbf{C})$. (The result of the division of $G L_{n}(\mathbf{C})$ by the product group can then be investigated afterward, one factor at a time.) If we arbitrarily choose an inner product on $\mathbf{C}^{n}$, say the standard one, then any invertible matrix $g \in G L_{n}(\mathbf{C})$ can be expressed in the polar decomposition $g=U h$, where $U$ is unitary and $h$ is a positive-definite Hermitian matrix (i.e. all its eigenvalues are strictly positive). This can be obtained from $h=\left(g^{\dagger} g\right)^{1 / 2}$, where the positive square root is taken for each positive eigenvalue of $g^{\dagger} g$, and $U=g h^{-1}$. The space of such $h$ is contractible; $h$ can be deformed to the identity $I$. Hence $G L_{n}(\mathbf{C})$ has a deformation retract [23] to the space $U(n)$ of unitary matrices.

For the second step, the group $G L_{n}(\mathbf{C}) / \mathbf{C}^{\times}$[where $\mathbf{C}^{\times}$ is embedded in $G L_{n}(\mathbf{C})$ as the subgroup of non-zero complex multiples of the identity] is also known as the projective linear group $P G L_{n}(\mathbf{C})$. It has a deformation retract onto $U(n) / U(1) \cong S U(n) / \mathbf{Z}_{n}$. Here $\mathbf{Z}_{n} \subset U(1) \subset U(n)$ is embedded in $S U(n)$ as the subgroup of $n \times n$ matrices that are a power of $\zeta$ times the identity; it is the center of $S U(n)$. (Note that, throughout the paper, we use $\cong$ to stand for topological isomorphism [homeomorphism] of topological spaces; when the space is also a group, the map is also an isomorphism of groups. In the special case of a discrete group, the discrete topology is used.)

Finally, we must also mod out by $\mathcal{J}_{n}$. It is a contractible subgroup of $G L_{n}(\mathbf{C})$, and intersects $\mathbf{C}^{\times} I$ only at $I$, so its image is still a contractible subgroup in $P G L_{n}(\mathbf{C})$. As for any space that is the quotient space $G / H$ of a group $G$ by a subgroup $H \subseteq G, P G L_{n}(\mathbf{C})$ can be viewed as a fibre bundle [23] over the quotient space $\mathcal{E P}_{n}$ with fibre $\cong \mathcal{J}$. Because the fibre is contractible, $\mathcal{E P}{ }_{n}$ has the same homotopy type as $P G L_{n}(\mathbf{C})$, or as $S U(n) / \mathbf{Z}_{n}$. That is, these spaces are homotopy equivalent, due to the existence of deformation retracts from $P G L_{n}(\mathbf{C})$ to $\mathcal{E P}{ }_{n}$, and from either of these to $S U(n) / \mathbf{Z}_{n}$.

The homotopy type of a space can also be studied by using the homotopy groups. We can apply the homotopy long exact sequence of a fibration to the fibre bundle $G$ over $G / H$ with fibre $H$ (where $H \subseteq G$ are groups) [23],

$\cdots \rightarrow \pi_{i+1}(G) \rightarrow \pi_{i+1}(G / H) \rightarrow \pi_{i}(H) \rightarrow \pi_{i}(G) \rightarrow \cdots$,

which holds down to $\pi_{0}(G / H)$ [for $i=0, \pi_{0}$ of a space is in general a set without a group structure, however for the present case the $\pi_{0} \mathrm{~s}$ are groups, except for $\pi_{0}(G / H)$ in the case that $H$ is not a normal subgroup of $G$ ]. If we apply this with $G=P G L_{n}(\mathbf{C})$ and $H=\mathcal{J}_{n}$, then as all homotopy groups (or sets) of $\mathcal{J}_{n}$ are zero (because it is contractible), we of course find again that $\pi_{i}\left(\mathcal{E P}_{n}\right) \cong$ $\pi_{i}\left(P G L_{n}(\mathbf{C})\right) \cong \pi_{i}\left(S U(n) / \mathbf{Z}_{n}\right)$ for all $i \geq 0$. Applying the sequence again with $G=S U(n), H=\mathbf{Z}_{n}$, and using (with $n \geq 2$ from here on) $\pi_{1}(S U(n))=\pi_{2}(S U(n))=0$, $\pi_{3}(S U(n)) \cong \mathbf{Z}$, and of course $\pi_{i}\left(\mathbf{Z}_{n}\right)=0$ for $i>0$, $\pi_{0}\left(\mathbf{Z}_{n}\right)=\mathbf{Z}_{n}$, we obtain $\left.\pi_{1}\left(\mathcal{E P} \mathcal{P}_{n}\right)=\mathbf{Z}_{n}, \pi_{2}(\mathcal{E P})_{n}\right)=0$,
$\pi_{3}\left(\mathcal{E} \mathcal{P}_{n}\right)=\mathbf{Z}$. For $n=2$, these results are well-known for $\mathcal{E} \mathcal{P}_{2} \cong \mathbf{R} \mathbf{P}^{3} \times \mathbf{R} \cong S O(3) \times \mathbf{R}$, and for general $n$ they are fairly well-known for $S U(n) / \mathbf{Z}_{n}$. We note that, when the fibre is a discrete group, these results can also be understood in terms of covering spaces; for example, $S U(n)$ is the simply-connected covering space of $S U(n) / \mathbf{Z}_{n}$, which means that $\pi_{1}\left(S U(n) / \mathbf{Z}_{n}\right) \cong \mathbf{Z}_{n}$.

Next we turn briefly to the homology and cohomology of $\mathcal{E} \mathcal{P}_{n}$, which depend only on the homotopy type of the space, and their applications (these are not essential for understanding the main text). The first homology group, with integer coefficients, is $H_{1}\left(\mathcal{E} \mathcal{P}_{n}\right) \cong \pi_{1}\left(\mathcal{E} \mathcal{P}_{n}\right) \cong \mathbf{Z}_{n}$ as $\pi_{1}\left(\mathcal{E} \mathcal{P}_{n}\right)$ is Abelian. By the universal coefficient theorem [23], the cohomology with integer coefficients is $H^{1}\left(\mathcal{E} \mathcal{P}_{n}\right)=0$, and $H^{2}\left(\mathcal{E P}{ }_{n}\right) \cong F_{2} \oplus T_{1}$, where $F_{2}$ is the free part (group of elements of infinite order) of $H_{2}\left(\mathcal{E P}_{n}\right)$, $T_{1}$ is the torsion part (group of elements of finite order) of $H_{1}\left(\mathcal{E P}_{n}\right)$, and the isomorphism is non-canonical. Thus $H^{2}\left(\mathcal{E} \mathcal{P}_{n}\right)$ contains a subgroup $\cong \mathbf{Z}_{n}$. For $n=2$, $H^{2}\left(\mathbf{R} \mathbf{P}^{3}\right) \cong \mathbf{Z}_{2}$, while $H^{3}\left(\mathbf{R} \mathbf{P}^{3}\right) \cong \mathbf{Z}$. For $n \geq 2$, using theorems of Hopf (Ref. [26], pp. 1-2, 41-42), there is a surjection from $\pi_{2}\left(\mathcal{E P}_{n}\right)=0$ onto $H_{2}\left(\mathcal{E P}_{n}\right)$, because the group homology $H_{2}\left(\pi_{1}\left(\mathcal{E} \mathcal{P}_{n}\right)\right)=H_{2}\left(\mathbf{Z}_{n}\right)=0$, and so $H_{2}\left(\mathcal{E} \mathcal{P}_{n}\right)=F_{2}=0$, implying $H^{2}\left(\mathcal{E P}_{n}\right) \cong \mathbf{Z}_{n}$. Alternatively, we can use a Cartan-Leray spectral sequence (see Ref. [26], p. 173 or Ref. [27], Sec. 6.10) to obtain $H^{2}\left(\mathcal{E} \mathcal{P}_{n}\right) \cong \mathbf{Z}_{n}$.

In general, the elements of the second integral cohomology group $H^{2}(X)$ for a topological space $X$ correspond one-to-one with the isomorphism classes of complex rank one vector bundles (also called line bundles) over $X$; the group operation corresponds to taking tensor products of line bundles. The element in $H^{2}(X)$ for a given line bundle is the first Chern class $c_{1}$ of the bundle; thus $c_{1}$ completely classifies line bundles up to isomorphism.

Our adiabatic transport construction does not directly produce a line bundle (because e.g. the dominant state depends on the path used), but the $g l_{1}(\mathbf{C})$ connection $n^{-1} \operatorname{tr} A$, or more precisely its holonomy around all possible loops, uniquely determines a line bundle (up to isomorphism), and hence determines its first Chern class $c_{1} \in H^{2}\left(\mathcal{E} \mathcal{P}_{n}\right) \cong \mathbf{Z}_{n}$. (Our connection is flat-see Sec. IIB 4 -but the statement would hold even if the connection were not flat; see e.g. Ref. [28], Sec. 2, for exposition and references.) Because our connection is flat, our formula Eq. (12) for the holonomy is precisely this $c_{1}$; when given a homotopy class of loops [in $\left.\pi_{1}\left(\mathcal{E} \mathcal{P}_{n}\right)\right]$, or the corresponding homology class of cycles [in $H_{1}\left(\mathcal{E} \mathcal{P}_{n}\right)$ ], it specifies an element of $\mathbf{Z}_{n}$, represented multiplicatively as a phase factor, which is the holonomy. Viewed as an element of $H^{2}\left(\mathcal{E} \mathcal{P}_{n}\right)$ (additively), $c_{1}$ is non-zero and in fact is a generator of $H^{2}\left(\mathcal{E} \mathcal{P}_{n}\right)$. This $c_{1}$ is an example of a torsion first Chern class, that is, one that is not equivalent to an integer or set of integers. 


\section{Appendix B: Gauge transformations and invariance of results}

The evolution equation (4) is covariant under $s$ dependent transformations lying in the subgroup $\mathbf{C}^{\times} \times$ $\mathcal{J}_{n}$. Precisely, if the column vector $|u\rangle$ is replaced by $\left|u^{g}\right\rangle=g|u\rangle$, where $g \in \mathbf{C}^{\times} \times \mathcal{J}_{n}$, then the evolution equation becomes $\varepsilon \partial_{s}\left|u^{g}\right\rangle=\left(J+\varepsilon A^{g}\right)\left|u^{g}\right\rangle$, where

$$
A^{g}=g A g^{-1}+\partial_{s} g \cdot g^{-1} \text {. }
$$

As $g$ is upper triangular, the inhomogeneous term $\partial_{s} g \cdot g^{-1}$ is as well (it is in the Lie algebra of $\mathbf{C}^{\times} \times \mathcal{J}_{n}$ ).

We show in Appendix $\mathrm{C}$ below that the leading terms $\propto \varepsilon$ in the coefficients $c_{i}$ in the characteristic equation contain elements of $A$ on or below the diagonal. It follows that the inhomogeneous terms in $A^{g}$ have no effect on the coefficients $c_{r}$ in order $\varepsilon$ except for the diagonal of $A^{g}$ which affects $c_{n-1} \propto \operatorname{tr} A$. Moreover, $J_{n}+\varepsilon g A g^{-1}=g\left(J_{n}+\varepsilon A\right) g^{-1}$ has the same eigenvalues as $J_{n}+\varepsilon A$. Hence the terms displayed in expression (77) are gauge invariant to the order shown, except for the $r=n$ term; the latter transforms as a $\mathbf{C}^{\times}$connection. Then the $s$ integrals of these terms are also gauge invariant, except that the Berry phase $(r=n)$ term changes by a multiple of $2 \pi i$ (where here $i$ is the square root of -1 , not an index), and thus is invariant except under a "large" gauge transformation, that is one that winds around the origin in $\mathbf{C}^{\times}$.

\section{Appendix C: Scaling of the characteristic equation and eigenvalues}

The characteristic equation of $J_{n}+\varepsilon A$ has the form

$$
\lambda^{n}+\sum_{i=0}^{n-1} c_{i} \lambda^{i}=0
$$

where the $c_{i}$ are similarity invariants of $J_{n}+\varepsilon A$, and all are of order $\mathcal{O}(\varepsilon)$ as $\varepsilon \rightarrow 0$. Indeed, the terms of first order in $\varepsilon$ in $c_{i}$ are $c_{i}=-\varepsilon \sum_{j=0}^{i} A_{n+j-i, 1+j}+\mathcal{O}\left(\varepsilon^{2}\right)$ for all $i=0, \ldots, n-1$.

Because any root of the equation must tend to zero as $\varepsilon \rightarrow 0$, it is not difficult to see that the $c_{0}$ term is the most important of the terms containing a $c_{i}$, provided that $\lim _{\varepsilon \rightarrow 0} c_{0} / \varepsilon \neq 0$. Then $\lambda \sim\left(-c_{0}\right)^{1 / n} \propto \varepsilon^{1 / n}$, and the other $c_{i}(i \neq 0)$ do not contribute at leading order in this limit.

\section{Appendix D: Generalized adiabatic theorem}

Here for completeness we prove the generalized version of the adiabatic theorem (including the Berry phase) in the context of the main text. The result is contained in Ref. [18], but our proof is different. We consider the evolution equation

$$
\partial_{s}|v\rangle=\left(\varepsilon^{-1} D+A^{\prime}\right)|v\rangle,
$$

where $D=\operatorname{diag}\left(\lambda_{0}, \ldots, \lambda_{n-1}\right)$ and $A^{\prime}$ is the Berry connection. In our case, $D$ consists of eigenvalues proportional to $\varepsilon^{1 / n}$ that are never equal, and $A^{\prime}$ has entries independent of $\varepsilon$. We assume that $\lambda_{0}$ has largest real part (i.e. it is dominant), and that the differences of the real parts of the $\lambda_{\mu} \mathrm{s}$ are bounded away from zero (this holds under the assumptions in the text). We also assume that all elements of $A^{\prime}$ are bounded in magnitude by the same constant $B>0$ uniformly for all $s$. First, we suppose that the system is prepared in the $\mu=0$ (dominant) eigenstate at $s=0$, and consider the amplitude for it to return to that eigenstate at $s=1$. If the possible transitions to other modes $(\mu \neq 0)$ are neglected, then the change in the complex amplitude of the dominant state will be a factor $\exp \left\{\int_{0}^{1} d s\left[\varepsilon^{-1} \lambda_{0}(s)+A_{00}^{\prime}(s)\right]\right\}$. We will show that corrections to this due to transitions, and the amplitude for ending in a different state, are of relative size $\mathcal{O}\left(\varepsilon^{[n-1] / n}\right)$ at most. We emphasize that our general argument applies whenever $D$ is diagonal and the differences of the real parts of the diagonal entries from the dominant one are bounded below by a non-zero constant times $\varepsilon$ to any power $<1$.

We first extract the factor $\exp \left\{\int_{0}^{1} d s\left[\varepsilon^{-1} \lambda_{0}(s)+\right.\right.$ $\left.\left.A_{00}^{\prime}(s)\right]\right\}$, to calculate relative to this expected factor; this has the effect of taking $\lambda_{0}=0$ (and $A_{00}^{\prime}=0$ ) without loss of generality, by subtracting these from the diagonal elements of $D$ (resp., $A^{\prime}$ ). Now we begin by considering $n=2$, and set $A_{11}^{\prime}=0$ for now. The change in amplitude is

$$
\left\langle v_{0}\left|\mathcal{P} \exp \int_{0}^{1} d s\left[\varepsilon^{-1} D+A^{\prime}\right]\right| v_{0}\right\rangle
$$

where the initial $\left|v_{0}\right\rangle=(1,0)^{T}$ is the dominant eigenstate of $J_{n}+\varepsilon A$ in this basis (and $\left\langle v_{0}\right|$ is the corresponding element of the dual basis). This transition amplitude is

$$
\begin{aligned}
= & \sum_{r=0}^{\infty} \int_{\mathcal{D}} \prod_{j=1}^{2 r} d s_{j} A_{01}^{\prime}\left(s_{2 r}\right) A_{10}^{\prime}\left(s_{2 r-1}\right) \cdots A_{10}^{\prime}\left(s_{1}\right) \\
& \times e^{\int_{s_{1}}^{s_{2}} \varepsilon^{-1} \lambda_{1}\left(s^{\prime}\right) d s^{\prime}+\cdots+\int_{s_{2 r-1}}^{s_{2 r}} \varepsilon^{-1} \lambda_{1}\left(s^{\prime}\right) d s^{\prime}},
\end{aligned}
$$

where $\mathcal{D}$ is the region defined by $s_{1}<s_{2}<\cdots<s_{2 r}$ and all $s_{j}$ in $[0,1]$. Subtracting the $r=0$ term (which is unity), and taking the absolute value, we have the bound

$$
\leq \sum_{r=1}^{\infty} \int_{\mathcal{D}} \prod_{j=1}^{2 r} d s_{j} B^{2 r} e^{-\varepsilon^{-1 / 2} L \sum_{j=1}^{r}\left(s_{2 j}-s_{2 j-1}\right)},
$$

where $\varepsilon^{1 / 2} L>0$ is a lower bound on $-\operatorname{Re} \lambda_{1}>0$ for all $s \in[0,1]$, which exists by our assumptions. [This expression, with 1 added, can be viewed as the partition function of a statistical mechanics problem of domain walls at positions $s_{j}$, where at $s=0,1$, the state vector is fixed at $\left|v_{0}\right\rangle$, and transitioning to the other state $\left|v_{1}\right\rangle=$ $(0,1)^{T}$ carries a fugacity $B$ and energy penalty $\varepsilon^{-1 / 2} L>$ 0 per unit length. As this penalty is large, domain walls 
are bound in pairs, and it is unlikely that state $\left|v_{1}\right\rangle$ is found.] This is in turn less than or equal to

$$
\leq \sum_{r=1}^{\infty} \frac{1}{r !} \int_{\mathcal{D}^{\prime}} \prod_{j=1}^{2 r} d s_{j} B^{2 r} e^{-\varepsilon^{-1 / 2} L \sum_{j=1}^{r}\left(s_{2 j}-s_{2 j-1}\right)}
$$

(where $\mathcal{D}^{\prime}$ is the domain $s_{1}<s_{2}, s_{3}<s_{4}, \ldots, s_{2 r-1}<$ $s_{2 r}$ and all $s_{j}$ in $\left.[0,1]\right)$, because the parts where some of the intervals $\left[s_{2 j-1}, s_{2 j}\right]$ overlap give positive contributions, and discarding these leaves a region that covers $\mathcal{D} r$ ! times. This multiple integral is a product, and gives rise to an exponential series with the initial term 1 omitted. Each two-dimensional integral factor can be evaluated to give $\varepsilon^{1 / 2} / L+\varepsilon\left(e^{-\varepsilon^{-1 / 2} L}-1\right) / L^{2}$, where the subleading terms are introduced by the integration limits at $s=0,1$. Hence we have found the upper bound

$$
=\exp \left[\varepsilon^{1 / 2} B^{2} / L+\varepsilon B^{2}\left(e^{-\varepsilon^{-1 / 2} L}-1\right) / L^{2}\right]-1 .
$$

As $\varepsilon \rightarrow 0$, this gives $\sim \varepsilon^{1 / 2} B^{2} / L$, which is simply the first term in the series.

In general, one should include $A_{11}^{\prime}$. This can be added to $\varepsilon^{-1 / 2} \lambda_{1}$, and can be absorbed into a change in the bound $L$ when $\varepsilon$ is sufficiently small. Hence the full result is

$$
\begin{aligned}
& \left\langle v_{0}\left|\mathcal{P} \exp \int_{0}^{1} d s\left[\varepsilon^{-1} D+A^{\prime}\right]\right| v_{0}\right\rangle= \\
& \exp \left\{\int_{0}^{1} d s\left[\varepsilon^{-1} \lambda_{0}(s)+A_{00}^{\prime}(s)\right]\right\} \cdot\left[1+\mathcal{O}\left(\varepsilon^{1 / 2}\right)\right]
\end{aligned}
$$

for the $n=2$ case. Similarly, we can show that the amplitude for making a transition to the state $\left|v_{1}\right\rangle$ during the evolution is

$$
\begin{aligned}
& \left\langle v_{1}\left|\mathcal{P} \exp \int_{0}^{1} d s\left[\varepsilon^{-1} D+A^{\prime}\right]\right| v_{0}\right\rangle= \\
& \mathcal{O}\left(\varepsilon^{1 / 2} B / L\right) \cdot \exp \left\{\int_{0}^{1} d s\left[\varepsilon^{-1} \lambda_{0}(s)+A_{00}^{\prime}(s)\right]\right\}
\end{aligned}
$$

as $\varepsilon \rightarrow 0$, and the same bound also applies to the amplitude for starting in 1 and ending in 0 .

Finally, for $n>2$ we can absorb $A_{\mu \mu}^{\prime}(\mu>0)$ into a change in the lower bound on all $-\operatorname{Re} \lambda_{\mu}, \mu \neq 0$. The remaining elements of $A_{\mu \nu}^{\prime}$ with $\mu, \nu \neq 0$, which produce transitions among those modes, are bounded in magnitude by $B$, and in the above argument simply lead to another order 1 contribution that can also be absorbed into $\varepsilon^{(1-n) / n} L$. Hence the result is similar, with corrections smaller at least by $\sim \varepsilon^{(n-1) / n} B^{2} / L$, and similarly for the amplitude for a transition to any state $\mu \neq 0$.
[1] T. Kato, Perturbation theory for linear operators (Springer, New York, 1980), Ch. 2.

[2] C. Miniatura, C. Sire, J. Baudon, and J. Bellissard, Europhys. Lett. 13, 199 (1990).

[3] W.D. Heiss, Phys. Rev. E 61, 929 (2000).

[4] C. Dembowski, H.-D. Gräf, H.L. Harney, A. Heine, W.D. Heiss, H. Rehfeld, and A. Richter, Phys. Rev. Lett. 86, 787 (2001).

[5] J. Doppler, A.A. Mailybaev, J. Böhm, U. Kuhl, A. Girschik, F. Libisch, T.J. Milburn, P. Rabl, N. Moiseyev, and S. Rotter, Nature 537, 76 (2016).

[6] T. Stehmann, W.D. Heiss and F.G. Scholtz, J. Phys. A 37, 7813 (2004).

[7] S.-B. Lee, J. Yang, S. Moon, S.-Y. Lee, J.-B. Shim, S.W. Kim, J.-H. Lee, and K. An, Phys. Rev. Lett. 103, 134101 (2009).

[8] Y. Choi, S. Kang, S. Lim, W. Kim, J.-R. Kim, J.-H. Lee, and K. An, Phys. Rev. Lett. 104, 153601 (2010).

[9] T. Gao, E. Estrecho, K.Y. Bliokh, T.C.H. Liew, M.D. Fraser, S. Brodbeck, M. Kamp, C. Schneider, S. Höfling, Y. Yamamoto, F. Nori, Y.S. Kivshar, A.G. Truscott, R.G. Dall, and E.A. Ostrovskaya, Nature 526, 554 (2015).

[10] R. Fleury, D.L. Sounas, and A. Alù, Nature Comm. 6, 5905 (2015).

[11] H. Xu, D. Mason, L. Jiang, and J.G.E. Harris, Nature 537, 80 (2016).

[12] Such subspaces can be accessed if there is complete control over the matrix elements of $H$, which can be achieved for $n=2$ in the optomechanical device demonstrated in
H. Xu, L. Jiang, A.A. Clerk, and J.G.E. Harris, Nature 568, 65 (2019).

[13] M. Born and V. Fock, Z. Phys. 51, 165 (1928).

[14] T. Kato, J. Phys. Soc. Jpn. 5, 435 (1950).

[15] M.V. Berry, Proc. Roy. Soc. A 392, 45 (1984).

[16] B. Simon, Phys. Rev. Lett. 51, 2167 (1983).

[17] F. Wilczek and A. Zee, Phys. Rev. Lett. 52, 2111 (1984).

[18] G. Nenciu and G. Rasche, J. Phys. A 25, 5741 (1992).

[19] R. Uzdin, A. Mailybaev, and N. Moiseyev, J. Phys. A 44, 435302 (2011).

[20] M.V. Berry and R. Uzdin, J. Phys. A 44, 435303 (2011).

[21] T.J. Milburn, J. Doppler, C.A. Holmes, S. Portolan, S. Rotter, and P. Rabl, Phys. Rev. A 92, 052124 (2015).

[22] A. Joye, Commun. Math. Phys. 275, 139 (2007).

[23] A. Hatcher, Algebraic Topology (Cambridge University, Cambridge, 2002).

[24] M. Naghiloo, M. Abbasi, Y.N. Joglekar, and K.W. Murch, Nat. Phys. 15, 1232 (2019).

[25] M. Aspelmeyer, T.J. Kippenberg, and F. Marquardt, Rev. Mod. Phys. 86, 1391 (2014).

[26] K.S. Brown, Cohomology of Groups, Graduate Texts in Mathematics 87 (Springer, New York, 1982).

[27] C. Weibel, Introduction to Homological Algebra (Cambridge University, Cambridge, 1995).

[28] D.S. Freed, G.W. Moore, and G. Segal, Annals Phys. 322, 236 (2007). 